\title{
Outcome of Pedicle Screw Fixation and Monosegmental Fusion in Patients with Fresh Thoracolumbar Fractures
}

\author{
Roop Singh ${ }^{1}$, Rajesh Kumar Rohilla ${ }^{1}$, Kulbhushan Kamboj ${ }^{1}$, Narender Kumar Magu ${ }^{1}$, Kiranpreet $\operatorname{Kaur}^{2}$ \\ ${ }^{1}$ Department of Orthopaedic Surgery, Paraplegia and Rehabilitation, PGIMS, Rohtak, India \\ ${ }^{2}$ Department of Anaesthesiology and Critical Care, PGIMS, Rohtak, India
}

\begin{abstract}
Study Design: Prospective clinical study.
Purpose: The present prospective study aims to evaluate the clinical, radiological, and functional and quality of life outcomes in patients with fresh thoracolumbar fractures managed by posterior instrumentation of the spine, using pedicle screw fixation and monosegmental fusion.

Overview of Literature: The goals of treatment in thoracolumbar fractures are restoring vertebral column stability and obtaining spinal canal decompression, leading to early mobilization of the patient.

Methods: Sixty-six patients (46 males and 20 females) of thoracolumbar fractures with neurological deficit were stabilized with pedicle screw fixation and monosegmental fusion. Clinical, radiological and functional outcomes were evaluated.

Results: The mean preoperative values of Sagittal index, and compression percentage of the height of the fractured vertebra were $22.75^{\circ}$ and 46.73 , respectively, improved (statistically significant) to $12.39^{\circ}$, and 24.91 , postoperatively. The loss of correction of these values at one year follow-up was not statistically significant. The mean preoperative canal compromise (\%) improved from $65.22 \pm 17.61$ to $10.06 \pm 5.31$ at one year follow-up. There was a mean improvement in the grade of 1.03 in neurological status from the preoperative to final follow-up at one year. Average Denis work scale index was 4.1. Average Denis pain scale index was 2.5. Average WHOQOL-BREF showed reduced quality of life in these patients. Patients of early surgery group (operated within 7 days of injury) had a greater mean improvement of neurological grade, radiological and functional outcomes than those in the late surgery group, but it was not statistically significant.

Conclusions: Posterior surgical instrumentation using pedicle screws with posterolateral fusion is safe, reliable and effective method in the management of fresh thoracolumbar fractures. Fusion helps to decrease the postoperative correction loss of radiological parameters. There is no correlation between radiographic corrections achieved for deformities and functional outcome and quality of life post spinal cord injury.
\end{abstract}

Keywords: Thoracolumbar; Fractures; Pedicle screws; Neurological involvement; Functional outcome; Radiological outcome

\section{Introduction}

The goals of treatment in thoracolumbar fractures are restoring vertebral column stability and obtaining spinal canal decompression, leading to early mobilization of the patient [1]. The appropriate treatment of thoracolumbar

Received Jan 9, 2013; Revised Mar 18, 2013; Accepted May 1, 2013

Corresponding author: Roop Singh

Department of Orthopaedic Surgery, Paraplegia and Rehabilitation,

52/9-J, Medical Enclave, PGIMS, Rohtak-124001, Haryana, India

Tel: +91-1262-213171, Fax: +91-1262-21308, E-mail: drroopsingh@rediffmail.com 
fractures remains a subject of controversy [2,3]. Different schools of thoughts for each modality, ranging from conservative management with reduction by pillow and extending today to anterior/posterior instrumentation systems for fixation, have proved that neither is an ideal method and much more needs to be done to reach an ideal modality of management $[2,4,5]$. Nonoperative treatment of fractures without neurologic deficits with bed rest, postural reduction, and bracing has been proposed, with variable results [4]. After conservative treatments, there are many reports on worsening spinal stenosis, increasing the pressure on the vertebral body and deteriorating the neurological functions. However, others who suggest surgical treatment, pointed that patients could expect to become mobile early, perform rehabilitative remedies, overcome anatomic fractures, restore swayback and improve, in most cases, the nervous functions by using decompression and fixation $[2,5]$. If surgical treatment of thoracolumbar fractures is chosen, further debate arises from the appropriate type of approach [6]. Anterior decompression and stabilization has been proposed for cases with severe narrowing of the spinal canal, complete comminution or dislocation, non-correctable deformities and mainly neurologic compromise. However, posterior approach is less extensive, and most spine surgeons advocate posterior fusion as the treatment of choice for unstable thoracolumbar injuries $[2,5,6]$.

Various modifications have been done in the posterior instrumentation system and technique. The transpedicular short-segment construct represents an attempt to restore the anterior column without the need for anterior strut grafting or plate fixation, avoiding extensive arthrodesis of the motion segments. The use of pedicle screws, which can minimize the range of spinal segments to reduce the damage to soft tissues and increase the rate of synostosis better than fixation using hooks and wires, expands the excellent functionalities of pedicle screws [6]. Although short-segment (SS) pedicle instrumentation has been considered as a superior method, several studies showed that SS pedicle instrumentation had a high rate of failure [3,7]. The parameters used for the evaluation of the final result (radiological, clinical and functional) have contributed to an increasing debate about the ideal method for the treatment of these fractures. Although some studies included clinical outcome measures, such as pain and functioning, many studies focused only on the radiological outcome $[1,3,7-9]$. In the present prospec- tive study, we aimed 1) to evaluate the overall clinical, radiological, and functional and quality of life (QOL) outcomes in patients with fresh thoracolumbar fractures managed by posterior instrumentation of the spine using the pedicle screw fixation and monosegmental fusion; 2) to find any correlation between radiographic corrections achieved after surgery and final outcomes of these parameters.

\section{Materials and Methods}

Sixty-six patients (46 males and 20 females) of acute spinal cord injury (SCI) with neurological deficit were presented to the author's institute, a tertiary level referral centre, between April 2007 and March 2011 and were included in the present prospective study. All patients who met the following eligibility criteria were included in the present study: 1) occurrence of a traumatic event resulting in unstable injury of the thoracolumbar spine with neurological deficit; 2) a minimum regular follow-up of 12 months; 3) signed informed consent; and 4) age older than 18 years. The criteria for the unstable spine injury were as follows: kyphotic deformity $(\mathrm{KD})>20^{\circ}$, progressively increasing neurological deficit, posterior ligamentous complex disruption, loss of vertebral body height by more than $50 \%$, and presence of free bony fragment within the spinal canal with incomplete neurological deficit.

The neurologically intact patients or patients with stable injury or with injury at multiple levels and those unfit for surgery/refusing surgery were excluded from the study. The mean age was $30.21 \pm 7.47$ years (range, 17-45 years). Thirty-eight patients had fallen from a height, 18 had road side accidents, and 10 experienced heavy objects falling on their back. Patients were given detailed information about the purpose of the study and written consent was obtained from all the participants. The complete history of patients was taken to rule out any other occult medical or neuropsychological problems and the complete general physical examination and neurological examination was done. Neurological deficit as per American Spinal Injury Association (ASIA) Impairment Scale were as follows: $\mathrm{A}$ in 34 ; $\mathrm{B}$ in 16 ; $\mathrm{C}$ in 12 ; $\mathrm{D}$ in 4 and $\mathrm{E}$ in none of the patients [10]. X-rays of the thoracolumbar spine, both anteroposterior (AP) and lateral views were done. A computed tomography (CT) scan was done to evaluate osseous injury, canal compromise, and to evaluate adjacent vertebral injury. Magnetic resonance 
imaging (MRI) was done to further observe the injury to the neurological structures and to determine the status of the posterior elements. Fifty-eight patients had single vertebral fracture and eight had two or more vertebral fractures. The levels of single vertebral fractures were D10 in 4 patients, D11 in 4, D12 in 12, L1 in 26, L2 in 8 and L3 in 4 patients. Eight patients who had two or more vertebrae fractures had levels between D9-L1. According to Denis classification, 42 patients had fracture dislocations and 24 patients had burst fractures [11]. As per thoracolumbar injury classification and severity score (TLICS), 2 patients had a score of 7, 54 had a score of 8 and 10 had a score of 9 [12]. Eight patients had fractures of calcaneum and ten patients had other associated skeletal injuries.

Patients and attendants were informed about the possible lines of the treatment. After the decision for surgical intervention was taken, patients were investigated as per the requirement of the preanesthetic check up. Surgery was performed as early as possible. Pedicle screw spinal system of rods and screws (both monoaxial and polyaxial versions) were used.

The mean time interval between injury and surgery was 13.1 days (range, 3-31 days). Patients were divided further in two groups for analysis. Group 1 included patients that were operated within 7 days (early surgery group, $\mathrm{n}=20$ ) and group 2 included patients that were operated after 7 days (late surgery group, $n=46$ ). The two groups were compared to evaluate whether timing of surgery had any effect on the final outcome.

A standard technique of pedicle screw insertion was used. Decompressive laminectomy at the fractured vertebral level was done in 24 patients with burst fractures showing severe canal stenosis on preoperative CT/MRI. Retropulsed fractured vertebral body was pushed anteriorly to decompress the spinal cord. Adequacy of the decompression was checked by looking for the pulsations in the dural sac, as well as by gently passing a blunt probe in the spinal canal of the superior and the inferior vertebrae. The status of the cord was noted whether contused, lacerated or completely transected. The nerve roots were identified and released from the compression, if present. Traumatic dural tear was observed in six cases and it was repaired. In one patient of fracture dislocation with complete translation of the spine, there was a complete transaction of the cord. Monosegmental bone grafts were put after decortication of transverse processes and facet joints of fractured vertebra by high speed drill to achieve local spine fusion. Fixation was done at 2 levels, one on either side of the fracture in 60 patients. Four level fixations were done (2 level fixations on either side) in 4 patients and 3 level fixations ( 2 levels proximal to fracture and 1 level distal to fracture) were done in 2 patients.

Early ambulation and rehabilitation was encouraged. Mobilisation and rehabilitation was done depending on the neurological recovery using different orthoses, e.g., spinal brace and knee-foot-ankle-orthoses. All patients were followed for a minimum of one year and the mean follow up was $24.3 \pm 8.7$ months (range, $12-39$ months). All patients were evaluated postoperatively at 1 month, 2 months, 4 months, 6 months, and 1 year. The parameters of radiographic evaluation were analyzed by the paired t-test and the chi-square test with Yates' correction. The correlation between the clinical, functional and radiologic results was calculated by Spearman correlation.

The preoperative measurements were compared with those of postoperative measurements, as well as followup measurements. The following radiological outcome measures were noted:

\section{1) $\mathrm{KD}$ (on sagittal view $\mathrm{X}$-rays) (Fig.1)}

Sagittal index (SI): SI was defined as the measurement of segmental kyphosis at the level of vertebra involved adjusted for the baseline sagittal contour at the level in the normal spine. It is calculated as the $\mathrm{KD}$ at the frac-

Diagram 1 - Kyphotic deformities

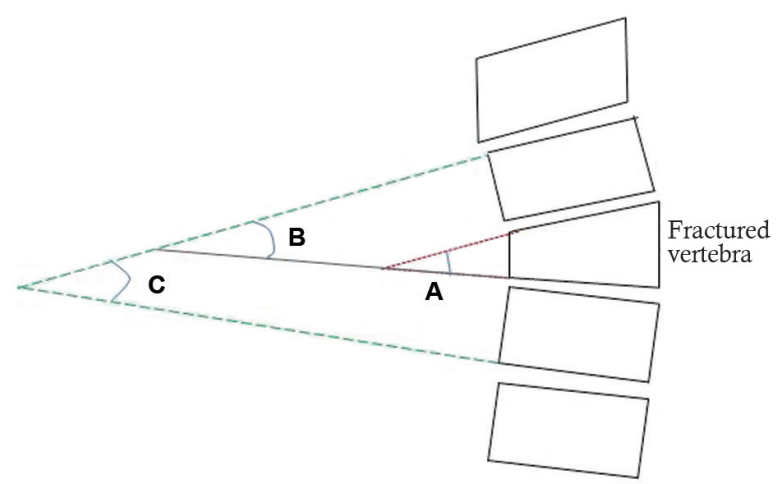

Angle $\mathrm{A}=$ Kyphotic deformity at fracture vertebra (KD)

Angle $B=$ Gardner's index (GI)

Angle $C=$ Regional kyphotic angle

Sagittal index $(\mathrm{SI})=\mathrm{KD}-\mathrm{NC}$, Where

$\mathrm{NC}=$ Normal kyphotic contour

Fig. 1. Diagram showing calculations of various kyphotic deformities. 
ture motion segment level minus the normal contour (NC) (SI=KD-NC). As an estimate of the baseline sagittal curve/level, an angle of $5^{\circ}$ was used in the thoracic segments, $0^{\circ}$ at the thoracolumbar junction, and $10^{\circ}$ in lumbar segments [13].

2) Compression percentage (CP) of the height of the fractured vertebra (Fig. 2): It was calculated by the formula $\mathrm{CP}=100-\mathrm{F} /[(\mathrm{A}+\mathrm{B}) / 2] \times 100$, where $\mathrm{F}$ is the anterior vertebra height at the fracture level; $A$ is the anterior vertebra height above the fracture level and $\mathrm{B}$ is the anterior vertebra height below the fracture level [13].

3) Canal dimensions were measured by a CT scan of the spine. Maximum AP diameter of the canal was measured in sagittal section at the level of the fractured vertebra. Canal compromise was expressed in percentage compromise; by taking the mean of the AP diameters of the canal in the two normal adjacent vertebrae (one on either of the fractured vertebrae) as normal canal diameter.

Neurological outcome was assessed after doing a neurological examination on each follow-up as per ASIA

\section{Diagram 2 - Compression percentage of} the fractured vertebral height

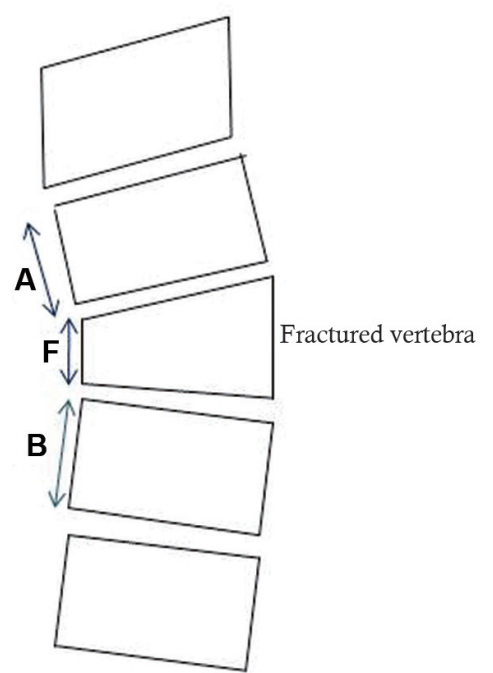

Compression percentage of the fractured vertebra1 height $(\mathrm{CP})=100-\mathrm{F} /[(\mathrm{A}+\mathrm{B}) / 2] \mathrm{X} 100$

$\mathrm{F}=$ anterior vertebral height at the fracture level $A=$ anterior vertebral height of upper vertebra $\mathrm{B}=$ anterior vertebral height of lower vertebra

Fig. 2. Diagram showing calculations of compression percentage of the fractured vertebral height. score. Functional outcomes were assessed according to Denis Pain Index [14], Denis Work Scale index [15], and Quality of life index (WHOQOL-BREF as defined by World Health Organization) [16].

\section{Results}

Table 1 shows various radiological parameters of the spine. The average preoperative SI of $22.75 \pm 8.29^{\circ} \mathrm{im}-$ proved to $12.39 \pm 6.68^{\circ}$ postoperatively. An average correction of $10.3^{\circ}$ was achieved for the SI, and there was an average loss of correction of $1.6^{\circ}$ in a six month follow-up period (SI, $14.0 \pm 6.61^{\circ}$ ) and $3.8^{\circ}$ in 1 year (SI, $\left.16.25 \pm 7.84^{\circ}\right)$. The correction was achieved from the preoperative to postoperative stage, in addition, a followup was significant $(p<0.001)$. The average compression percentage was $46.73 \pm 13.89 \%$ preoperatively. It was improved to $24.91 \pm 10.64 \%$ postoperatively, decreased to $27.23 \pm 11.45 \%$ at 6 months and $29.66 \pm 14.37 \%$ at 1 year. The improvement achieved from the preoperative to postoperative stage, as well as at the follow-up was significant $(p<0.001)$. There was no correlation between the correction loss during the follow up and the initial deformity for SI and percentile vertebral compression. The average canal compromise was $65.22 \pm 17.61 \%$ preoperatively. It was improved to $12.67 \pm 7.31 \%$ (by $52 \%$ ) at 6 months follow-up and $10.06 \pm 5.31 \%$ (by $55 \%$ ) at 1 year (Table 1). The improvement was significant for both at 6 months, as well as 1 year $(p<0.001)$. There was no statistical significant difference in the final values of the SI and compression percentage of the vertebral height between patients in whom laminectomy was done $(n=24)$ and those without laminectomy $(\mathrm{n}=42)$. However, patients with laminectomy had better canal clearance $(p<0.01)$.

There was an improvement of average 1.03 grades in neurological status as per ASIA Impairment scale from the preoperative to final follow-up at one year (Table 2). The neurological recovery was highly significant $(p=0.000$ using chi-square test with Yates' correction). Eighteen (27\%) patients did not improve, 34 patients (51\%) improved by one grade, 8 patients (12\%) improved by two grades and 6 patients (9\%) improved by three grades. Twelve patients (18.2\%) improved fully to grade E. A total of $41 \%$ patients of the preoperative grade A improved by one or more grades. All patients who did not improve had complete paraplegia (ASIA A) at the time of injury.

Average Denis work scale index was 4.1. Six patients 
Table 1. Various radiological outcome measures

\begin{tabular}{lcccc} 
& Initial & Postoperative & 6 mo & $1 \mathrm{yr}$ \\
Sagittal index $\left(^{\circ}\right)$ & $22.75 \pm 8.29$ & $12.39 \pm 6.68$ & $14.00 \pm 6.61$ & $16.25 \pm 7.84$ \\
Compression percentage of vertebral height & $46.73 \pm 13.89$ & $24.91 \pm 10.64$ & $27.23 \pm 11.45$ & $29.66 \pm 14.37$ \\
Canal compromise $(\%)$ & $65.22 \pm 17.61$ & - & $12.67 \pm 7.31$ & $10.06 \pm 5.31$ \\
\hline
\end{tabular}

Table 2. Change in neurological status

\begin{tabular}{lccccc} 
& \multicolumn{5}{c}{ Follow-up at one year } \\
\cline { 2 - 6 } Preoperative & A & B & C & D & E \\
A $(n=34)$ & 18 & 14 & - & 4 & - \\
B $(n=16)$ & - & - & 8 & 8 & 4 \\
$C(n=12)$ & - & - & - & - & 4 \\
$D(n=4)$ & - & - & 8 & 14 & 4 \\
\hline Total & 18 & 14 & - & 12 \\
\hline
\end{tabular}

Table 3. Radiological parameters in early and late surgery group

\begin{tabular}{|c|c|c|c|c|c|c|c|c|}
\hline & \multicolumn{4}{|c|}{ Operated within 7 days group $1(n=20)$} & \multicolumn{4}{|c|}{ Operated $>7$ days group 2 ( $n=46)$} \\
\hline & Initial & Postoperative & $6 \mathrm{mo}$ & $1 \mathrm{yr}$ & Initial & Postoperative & $6 \mathrm{mo}$ & $1 \mathrm{yr}$ \\
\hline Sagittal index & $23.70 \pm 9.25$ & $9.80 \pm 5.98$ & $12.30 \pm 7.17$ & $14.50 \pm 8.02$ & $22.35 \pm 8.04$ & $13.52 \pm 6.78$ & $14.74 \pm 6.38$ & $17.30 \pm 7.97$ \\
\hline $\begin{array}{c}\text { Compression } \\
\text { percentage }\end{array}$ & $50.50 \pm 16.11$ & $23.90 \pm 13.63$ & $24.60 \pm 12.60$ & $27.50 \pm 16.01$ & $45.09 \pm 12.86$ & $25.35 \pm 9.38$ & $28.17 \pm 11.04$ & $30.60 \pm 14.07$ \\
\hline Canal compromise & $59.10 \pm 25.81$ & - & $13.60 \pm 9.19$ & $12.67 \pm 6.89$ & $67.87 \pm 12.43$ & - & $12.26 \pm 6.53$ & $8.10 \pm 3.48$ \\
\hline
\end{tabular}

returned to the previous level of work. Six patients returned to full time work with mild restriction. The rest did not return to their previous work level. Forty-two (63.64\%) were completely disabled. Most of these initially had complete injury. The average Denis pain scale index was 2.5 . Four patients $(6.06 \%)$ were in grade 1 (no pain); $28(42.42 \%)$ in grade 2 (occasional, minimal pain, no need for medication); 28 (42.42\%) in grade 3 (moderate pain, occasional medication, no interruption of work or activities of daily livings); 4 (6.06\%) in grade 4 (moderate to severe pain, occasional absences from work, significant in activities of daily livings); and 2 patients (3.03\%) were in grade 5 (constant severe pain, chronic medication). The average WHOQOL-BREF Domain 1 score was 41.33; Domain 2 score was 40.75; Domain 3 score was 45.63; and Domain score 4 was 51.21. There was no correlation between the radiographic corrections achieved for deformities and functional outcome and QOL post-SCI.

Patients of the early surgery group (group 1) improved by an average of 1.2 grades of ASIA grading, while pa- tients of the late surgery group (group 2) improved by an average of 0.95 grades. Improvement was statistically significant in both groups $(p<0.01)$. The mean improvement was slightly more in group 1 than group 2 , but the difference between the two groups was not statistically significant. There was significant improvement in the radiological and functional parameters from preoperative to postoperative, and on follow-ups in both groups $(p<0.01)$ (Table 3$)$ with slightly more improvement in group 1 than in group 2 .

One patient had implant failure as nut loosening, noticed on X-ray incidentally 20 months after the surgery. The patient recovered fully and was asymptomatic. Four screws (in 3 patients) were misplaced as evident from postoperative radiographs. None of these had any complications until the final follow-up.

Figs. 3, 4 are the preoperative and postoperative $\mathrm{X}$ rays, CT scan and MRI photographs of the representative cases. 


\section{Discussion}

Most authors believe that surgical treatment is needed for unstable fractures, the choice for operative approaches remains disputed $[2,6]$. Common opinion is to obtain the most stable fixation by fixing as few vertebrae as possible with neural canal decompression. SS posterior fixation is the most common and simple treatment, offering the advantage of incorporating fewer motion segments in the fusion $[2,5,6]$. However, several studies showed that SS pedicle instrumentation had a high rate of failure [3,7], and many studies have focused on the radiological outcome only $[1,3,7-9]$. The present study aimed to evaluate the short segment pedicle screw fixation in relation to clinical, radiological, as well as functional criteria.

The mean time interval between the injury and the sur- gery was 13.1 days (range, 3-31 days) primarily because we do not have the facilities for emergency stabilization of the spine, and secondly, mostly patients reported late either due to referral from peripheral centres or ignorance on the part of the patient. Similar time interval was reported by the other studies from Indian subcontinent $[7,8]$. Early stabilization of thoracolumbar spinal fractures favors neurological improvement [17]. Although highest recovery rates had been reported for patients operated on within 48 hours of the initial trauma [17], but even if surgery was delayed, there were fair chances of neurological recovery and correction of deformity $[7,8]$. We observed the improvement of average 1.03 grade in a neurological status as per ASIA grading from preoperative to final a follow-up, despite the high injury-surgery interval. Chadha and Bahadur [8] and Butt et al. [7] had also re-
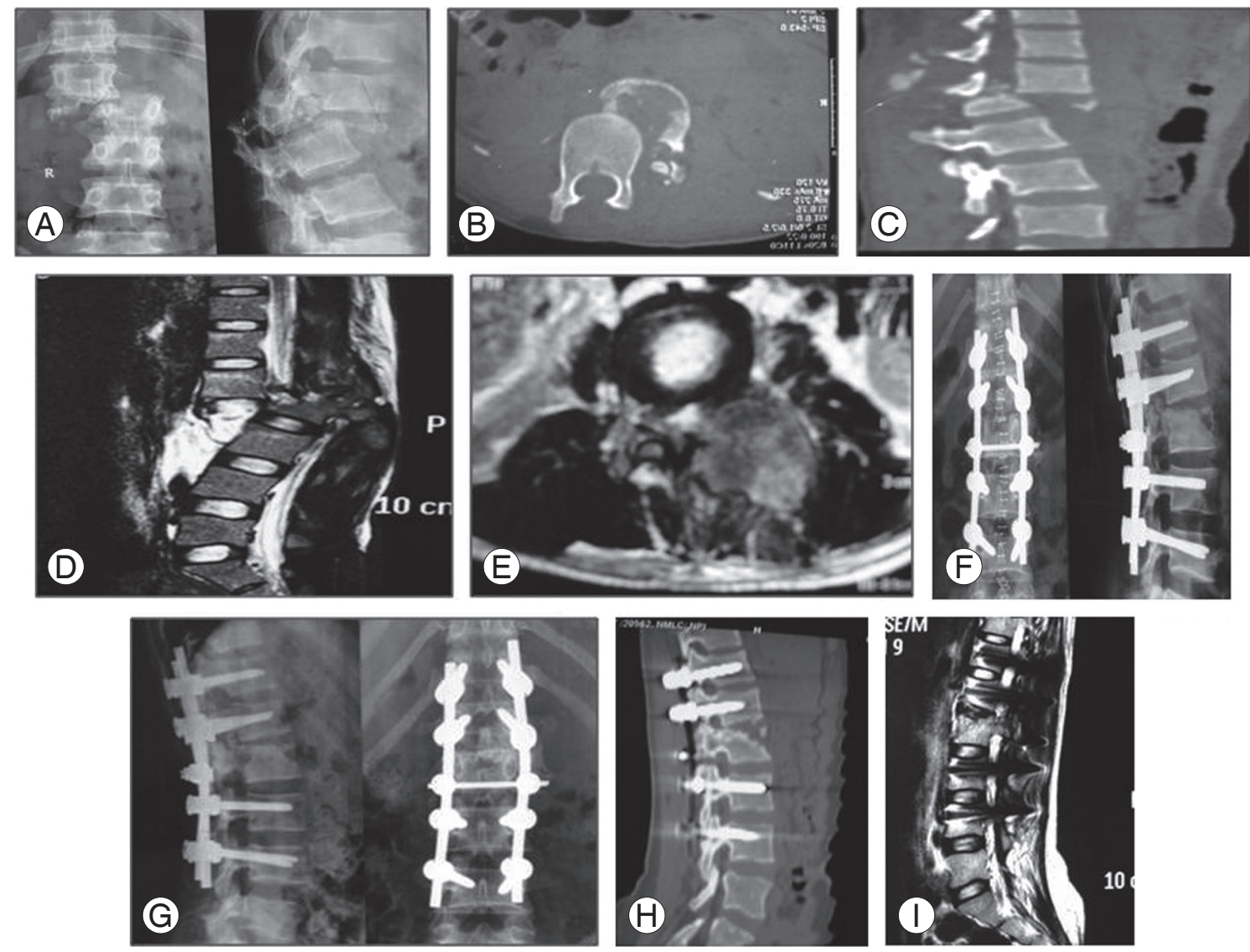

Fig. 3. L1/L2 fracture dislocation in a 35-year-old female as a result of fall of a heavy object while doing agriculture work. Patient was complete paraplegic. Fracture type: Denis-fracture dislocation of L1 over L2, thoracolumbar injury classification and severity score (TLICS) score-8, load-sharing classification score-8. (A) Anteroposterior and lateral radiograph. Preoperative thoracolumbar spine computed tomography (CT) axial (B) and sagittal (C) images. Preoperative thoracolumbar spine magnetic resonance imaging (MRI) sagittal (D) and axial (E) images. (F) Postoperative anteroposterior and lateral radiographs. (G) One year follow-up anteroposterior and lateral radiograph. One year follow-up thoracolumbar spine CT sagittal (H) and MRI sagittal (I) images. Healing in normal alignment is seen. Patient had complete transaction of the cord. 
ported a fair neurological recovery in delayed surgery cases. In the present study, the patients of early surgery group showed more improvement of the average ASIA score (1.2 grade) than the patient of late surgery group (0.95 grade), but the difference was not statistically significant $(p=0.966)$. We also observed neurological improvement in patients even when they were operated as late as 4 weeks. We are of the opinion that there are fair chances of neurological recovery, even when surgery is delayed. In the present study, the patients having incomplete lesions of the spinal cord showed more neurological improvement (average, 1.5 grade) than the complete lesion of the spinal cord (average, 0.53 grade). Similar observations were reported in other studies $[5,7,18]$. Patients who did not improve were those with complete paraplegia (ASIAA) at the time of injury. This indicates that the initial clinical state of massive and complete neural injury have an unfavorable prognosis. There was no correlation between most of the radiographic values (correction achieved) and neurological recovery in the present study. Similar results were shown by other studies $[2,5,9,15]$. Thus, the factors predicting a neurological prognosis for the fractures of the thoracolumbar spine appear to be complex and initial clinical state of neurological structures appears to be of a more prognostic value.

The improvements in SI in the present study were comparable to those reported in literature [2,3,5,7,9,19-21]. The corrections achieved from preoperative to postoperative stage, as well as the follow-up were highly significant $(p<0.001)$. Many studies reported statistically significant loss of achieved corrections on the follow-up $[1,7,8]$. The reported mean correction loss ranged from $0.3^{\circ}$ to $15.4^{\circ}$ in various studies $[1,3,5,7,15,22-24]$. We had less loss of correction probably because we had done posterolateral fusion in all patients. Wang et al. [18] also reported a less loss of correction in sagittal curve in the fusion group;
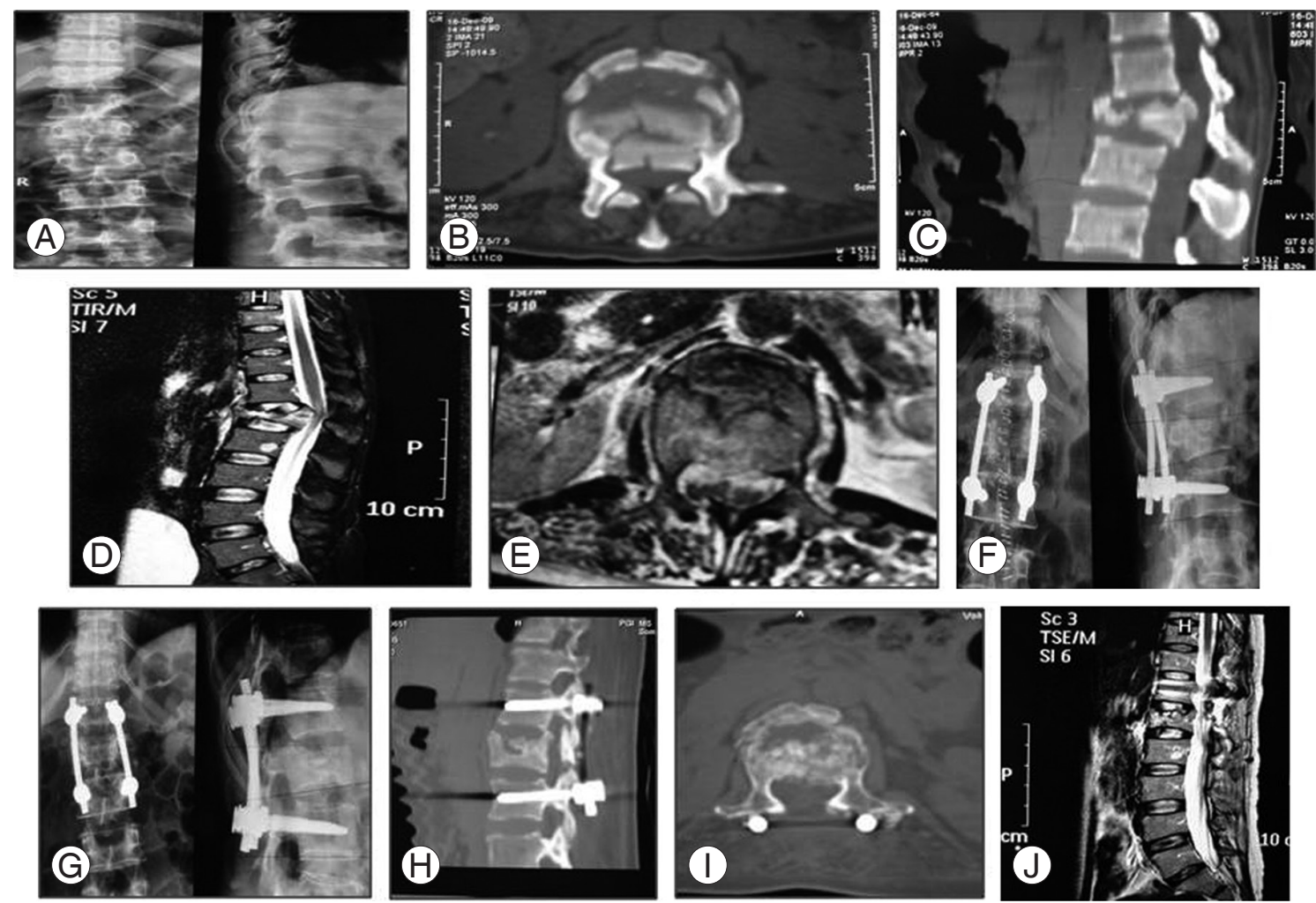

Fig. 4. L1 burst fracture in a 45-year-old male as a result of traffic accident. Patient had American Spinal Injury Association (ASIA)-C grade neurological involvement. Fracture type: Burst fracture, thoracolumbar injury classification and severity score (TLICS) score-8, load-sharing classification score-7. (A) Anteroposterior and lateral radiograph. Preoperative thoracolumbar spine computed tomography axial (B) and sagittal (C) images. Preoperative thoracolumbar spine magnetic resonance imaging (MRI) sagittal (D) and axial (E) images. Preoperative computed tomography (CT) and MRI show 60\% canal compromise. (F) Postoperative anteroposterior and lateral radiographs. (G) One year follow-up anteroposterior and lateral radiograph. There is $5^{\circ}$ correction loss. One year followup thoracolumbar spine computed tomography sagittal (H) and axial (I) and MRI sagittal (J) images. Follow-up CT and MRI show complete restoration of the canal. Healing in normal alignment is seen. Patient had complete neurological recovery. 
although there was no statistically significant difference between the fusion and non-fusion groups. We did not encounter any major loss of corrected deformity $\left(>10^{\circ}\right)$ in the present study. Some of the studies had reported correction loss $>10^{\circ}$ after short segment posterior instrumentation [24]. The postoperative loss of achieved correction had been attributed to the changes in adjacent disc space $[1,22]$. In the present study, we did not observe any correlation between the correction loss and the initial deformity for SI (Spearman's correlation coefficient, 0.003; significance [2-tailed], 0.991; significant at 0.01 level).

The application of posterior fusion techniques to the short segment fixation in cases of trauma remains controversial [25-27]. Qian et al. [26] reported that the posterolateral fusion was an effective measure to prevent implant failure, but Sanderson et al. [25] and Dai et al. [27] recommended that routine fusion was unnecessary in the operative management of these fractures. We had less loss of the postoperative correction achieved for all radiological parameters on the final follow-up as compared to the literature $[5,7]$. We are of the opinion that the posterolateral fusion is advantageous in posterior fixation of thoracolumbar fractures surgery.

The improvement in average compression percentage of the fractured vertebra in the present study was comparable to the correction achieved in most of the previous studies [3,7,9,19-21]. Although there was $20.6 \%$ loss of correction on the final follow-up, the overall final correction achieved was significant $(p<0.001)$. Other studies also reported an average loss of $2.7 \%-10 \%$ of preoperative value $[3,5,9]$. In the present study, we did not observe any correlation between the loss of correction and the initial compression percentage (Spearman's correlation coefficient, 0.032; significance [2-tailed], 0.081; significant at 0.01 level).

We had performed laminectomy and canal decompression in $36.3 \%$ of the patients who had initial severe canal compromise (average canal compromise, 65.22\%), i.e., grade 3 as per Weyns et al. Weyns et al. [28] grading. They had also favoured laminectomy in patients with severe canal compromise [28]. By doing a laminectomy, we could visualize the status of the cord, any dural tear and nerve root entrapment. It helped us in recognizing and repairing the dural tears in six patients and removing intradural piece of the bone in one patient. We also observed improvement of the canal diameter after six months, up to one year, which indicated spontaneous canal remodelling with time, as also observed by another study [29]. There was no correlation between the initial, as well as of the final canal compromise with neurological recovery. Similar observations were reported by Herndon and Galloway [30]. This indicates that bony canal dimension improvement alone does not guide to a favourable outcome; initial trauma to neural tissue and vitality of neural structures is of more significance. The present study also demonstrates that laminectomy does not lead to instability in short segment fixations if posterolateral fusion is also done simultaneously (no statistical significant difference in Saggital index between two groups of patients). However, it helps in better canal clearance $(p<0.01)$.

The average Denis pain scale index was 2.5. Similar results were shown by previous studies [3,5,7,9]. Only $20 \%$ of the patients were able to return to their previous job as per Denis work scale index in the present study, and most of the incompletely recovered patients required job modifications. Leferink et al. [22] also reported that $50 \%$ of patients had to change the intensity of their work or the kind of work after thoracolumbar spine injury and treatment. Other studies had also reported a low working ability after thoracolumbar spine fractures [31]. There was no correlation between Denis work scale index and pain scale index with the radiographic correction achieved. Knop et al. [1] also observed no correlation between the Hannover spine score and conventional radiographic, as well as CT findings. This indicates that radiological correction of $\mathrm{KD}$ alone does not improve functional outcome and functional recovery is more dependent on neurological recovery.

Assessment of the general health related QOL has now become an important integral part in evaluating the success of a specific medical treatment [31]. However, so far, only a very few data exist reflecting the QOL after thoracolumbar fractures [31]. We observed a lower post injury QOL. Briem et al. [31] also reported a lower QOL compared to healthy controls after thoracolumbar spine fractures. They reported that the factors like pain acceptance/ pain depression, compensation, patient's coping strategies, such as hypochondriasis, and general satisfaction etc. may influence QOL and functional outcomes after thoracolumbar injury. Knop et al. [1] also reported that the goal of fully functional integration of all patients back into society was difficult. We observed no correlation between most of the radiographic correction achieved 
and the functional results. Leferink et al. [22] also did not observe any correlation between the anterior wedge angle and regional angle on one hand and functional capacity tests on the other hand. Sanderson et al. [25] also reported that the lack of correlation of clinical results with bony radiographic parameters and highlighted the likely importance of the associated soft tissue on the outcome. This feature was usually ignored in the literature as it was very difficult to quantify and measure [25]. The results observed in the present study suggest that other still undefined parameters along with psychosocial status influence the functional results for thoracolumbar spine fractures.

There are many reports of implant failure with short segment pedicle screw fixation $[7,8]$. In the study by Butt et al. [7], more than $50 \%$ of patients had one or more complications, including 18 cases of hardware failures, involving 20 pedicle screws. We had only one implant failure as the nut loosening that also noticed after 20 months of surgery on X-ray incidentally and patient remained asymptomatic. We had no screw bending or breakage probably fusion protected the implant from fatigue $[18,25]$. Moreover, patients were advised strict wearing of spinal brace for the initial 2-3 months. Four of our screws (in 3 patients) as evident from postoperative radiographs, were misplaced. Chadha and Bahadur [8] reported misplacement of the screw in 3 cases, one screw pullout and one case of screw loosening. A good quality image intensifier to know the intraoperative positioning of the screws is a must for good results. Our policy of preoperative CT scan measurements of pedicle diameter and angulations might be the reason for less malpositioning of the screws. All patients with malpositioned screw(s) were asymptomatic. It may be due to our more cautious approach in their rehabilitation, as they were advised to strictly wear spinal brace for a longer period (5-6 months). We did not routinely remove the implants. Sanderson et al. [25] also reported that due to the lack of complications of implant at 2 years, routine removal of implant might not be necessary.

The study has limitation that there are few cases of 4 level or 3 level fixation so we could not compare these cases with two level fixations. All the patients had neurological deficit (majority of the patients were in ASIA A grade); therefore, we could also not evaluate the difference between neurologically intact and neurologically deficit patients. Future studies taking these aspects into consideration may help to better understand the difference in these groups of patients.

\section{Conclusions}

From this prospective study, we conclude that posterior surgical instrumentation using pedicle screws with posterolateral fusion is safe, reliable and effective method in the management of thoracolumbar fractures. It helps in attaining the goal of restoration of dynamic and protectivefunction of the spine, in creating optimum condition for an eventual recovery of the neurological structures, and functional outcome. Addition of posterolateral fusion helps in the prevention of implant failure, loss of achieved correction and post traumatic kyphosis. There is no correlation between the correction loss during the follow up and the initial deformity for SI and percentile vertebral compression; and also between radiographic corrections achieved for deformities and functional outcome and QOL post-SCI.

\section{Conflict of Interest}

No potential conflict of interest relevant to this article was reported.

\section{References}

1. Knop C, Fabian HF, Bastian L, et al. Fate of the transpedicular intervertebral bone graft after posterior stabilisation of thoracolumbar fractures. Eur Spine J 2002;11:251-7.

2. Yue JJ, Sossan A, Selgrath C, et al. The treatment of unstable thoracic spine fractures with transpedicular screw instrumentation: a 3-year consecutive series. Spine (Phila Pa 1976) 2002;27:2782-7.

3. Modi HN, Chung KJ, Seo IW, et al. Two levels above and one level below pedicle screw fixation for the treatment of unstable thoracolumbar fracture with partial or intact neurology. J Orthop Surg Res 2009; 4:28.

4. Dai LY, Jiang LS, Jiang SD. Conservative treatment of thoracolumbar burst fractures: a long-term follow-up results with special reference to the load sharing classification. Spine (Phila Pa 1976) 2008;33:2536-44.

5. Shin TS, Kim HW, Park KS, Kim JM, Jung CK. Shortsegment pedicle instrumentation of thoracolumbar 
burst-compression fractures: short term follow-up results. J Korean Neurosurg Soc 2007;42:265-70.

6. Kothe R, Panjabi MM, Liu W. Multidirectional instability of the thoracic spine due to iatrogenic pedicle injuries during transpedicular fixation. A biomechanical investigation. Spine (Phila Pa 1976) 1997; 22:1836-42.

7. Butt MF, Farooq M, Mir B, Dhar AS, Hussain A, Mumtaz M. Management of unstable thoracolumbar spinal injuries by posterior short segment spinal fixation. Int Orthop 2007;31:259-64.

8. Chadha M, Bahadur R. Steffee variable screw placement system in the management of unstable thoracolumbar fractures: a Third World experience. Injury 1998;29:737-42.

9. Defino HL, Canto FR. Low thoracic and lumbar burst fractures: radiographic and functional outcomes. Eur Spine J 2007;16:1934-43.

10. Maynard FM Jr, Bracken MB, Creasey G, et al. International standards for neurological and functional classification of spinal cord injury. American Spinal Injury Association. Spinal Cord 1997;35:266-74.

11. Denis F. Spinal instability as defined by the threecolumn spine concept in acute spinal trauma. Clin Orthop Relat Res 1984;(189):65-76.

12. Vaccaro AR, Zeiller SC, Hulbert RJ, et al. The thoracolumbar injury severity score: a proposed treatment algorithm. J Spinal Disord Tech 2005;18:209-15.

13. Farcy JP, Weidenbaum M, Glassman SD. Sagittal index in management of thoracolumbar burst fractures. Spine (Phila Pa 1976) 1990;15:958-65.

14. Denis F, Armstrong GW, Searls K, Matta L. Acute thoracolumbar burst fractures in the absence of neurologic deficit. A comparison between operative and nonoperative treatment. Clin Orthop Relat Res 1984;(189):142-9.

15. Liu S, Li H, Liang C, et al. Monosegmental transpedicular fixation for selected patients with thoracolumbar burst fractures. J Spinal Disord Tech 2009; 22:38-44.

16. Development of the World Health Organization WHOQOL-BREF quality of life assessment. The WHOQOL Group. Psychol Med 1998;28:551-8.

17. Kennedy JG, Soffe KE, McGrath A, Stephens MM, Walsh MG, McManus F. Predictors of outcome in cauda equina syndrome. Eur Spine J 1999;8:317-22.

18. Wang ST, Ma HL, Liu CL, Yu WK, Chang MC, Chen
TH. Is fusion necessary for surgically treated burst fractures of the thoracolumbar and lumbar spine?: a prospective, randomized study. Spine (Phila Pa 1976) 2006;31:2646-52.

19. Kim HS, Lee SY, Nanda A, et al. Comparison of surgical outcomes in thoracolumbar fractures operated with posterior constructs having varying fixation length with selective anterior fusion. Yonsei Med J 2009;50:546-54.

20. Ekapichon S. Intermediate screws in short segment pedicular fixation for thoracolumbar and lumbar burst fractures. Thai J Orthop Surg 2009;33:10-5.

21. Gelb D, Ludwig S, Karp JE, et al. Successful treatment of thoracolumbar fractures with short-segment pedicle instrumentation. J Spinal Disord Tech 2010;23: 293-301.

22. Leferink VJ, Keizer HJ, Oosterhuis JK, van der Sluis $\mathrm{CK}$, ten Duis HJ. Functional outcome in patients with thoracolumbar burst fractures treated with dorsal instrumentation and transpedicular cancellous bone grafting. Eur Spine J 2003;12:261-7.

23. Leferink VJ, Zimmerman KW, Veldhuis EF, ten Vergert EM, ten Duis HJ. Thoracolumbar spinal fractures: radiological results of transpedicular fixation combined with transpedicular cancellous bone graft and posterior fusion in 183 patients. Eur Spine J 2001;10:517-23.

24. Altay M, Ozkurt B, Aktekin CN, Ozturk AM, Dogan O, Tabak AY. Treatment of unstable thoracolumbar junction burst fractures with short- or long-segment posterior fixation in magerl type a fractures. Eur Spine J 2007;16:1145-55.

25. Sanderson PL, Fraser RD, Hall DJ, Cain CM, Osti OL, Potter GR. Short segment fixation of thoracolumbar burst fractures without fusion. Eur Spine J 1999;8:495-500.

26. Qian BP, Qiu Y, Wang B, Yu Y, Zhu ZZ. Effect of posterolateral fusion on thoracolumbar burst fractures. Chin J Traumatol 2006;9:349-55.

27. Dai LY, Jiang LS, Jiang SD. Posterior short-segment fixation with or without fusion for thoracolumbar burst fractures: a five to seven-year prospective randomized study. J Bone Joint Surg Am 2009;91:103341.

28. Weyns F, Rommens PM, Van Calenbergh F, Goffin J, Broos P, Plets C. Neurological outcome after surgery for thoracolumbar fractures. A retrospective study of 
93 consecutive cases, treated with dorsal instrumentation. Eur Spine J 1994;3:276-81.

29. Leferink VJ, Nijboer JM, Zimmerman KW, Veldhuis EF, ten Vergert EM, ten Duis HJ. Burst fractures of the thoracolumbar spine: changes of the spinal canal during operative treatment and follow-up. Eur Spine J 2003;12:255-60.
30. Herndon WA, Galloway D. Neurologic return versus cross-sectional canal area in incomplete thoracolumbar spinal cord injuries. J Trauma 1988;28:680-3.

31. Briem D, Behechtnejad A, Ouchmaev A, et al. Pain regulation and health-related quality of life after thoracolumbar fractures of the spine. Eur Spine J 2007;16:1925-33. 